\title{
Una experiencia de aprendizaje-servicio en la asignatura «Bases anatómicas y fisiológicas del movimiento» del Área de Didáctica delaExpresión Corporal A service-learning experience in the subject «Anatomical and physiological bases of movement» belonging to the Didactic of Corporal ExpressionArea
}

\author{
Jesús Gil Gómez, Óscar Chiva Bartoll \\ Universitat Jaume I de Castellón
}

\begin{abstract}
Resumen: El presente artículo expone una experiencia innovadora de aprendizaje-servicio diseñada y aplicada en el Área de Didáctica de la Expresión Corporal de la Universitat Jaume I. Concretamente se trata de una aplicación basada en el documento «Estándares de Aprendizaje-Servicio K-12 para la Práctica de Calidad» propuesto en 2008 por el National Youth Leadership Council de Estados Unidos. En esta aplicación didáctica han intervenido, por una parte, el alumnado de la asignatura «Bases anatómicas y fisiológicas del movimiento», perteneciente a la titulación de maestro y maestra de la Universitat Jaume I, y por otra parte, varias entidades sociales centradas en la atención a personas con dificultades motrices derivadas de diversas patologías anatómicas y/o fisiológicas. A través de esta metodología el alumnado universitario ha aplicado los conocimientos específicos de la asignatura en cuestión, para prestar un servicio a la comunidad. Así, no sólo ha aprendido los contenidos curriculares pertinentes de un modo práctico y significativo, sino que además ha puesto en juegosu competencia social y ciudadana. En definitiva el artículo recoge, una a una, las diferentes fases llevadas a cabo en esta experiencia de aprendizaje-servicio en el ámbito de la educación física: el diseño, la aplicación práctica y la valoración final de la misma.

Palabras clave: Aprendizaje-Servicio, Didáctica de la Educación Física, Innovación Metodológica, Diversidad Funcional, Educación Superior.
\end{abstract}

\begin{abstract}
This paper presents the design and implementation of an innovative service-learning experience carried out in the Physical Education Department of the Universitat Jaume I. The experience is based in the «K -12 Service -Learning Standards for Quality Practice» proposed in 2008 by the National Youth Leadership Council of the U.S. In this didactic application there were involved different groups of people. On the one hand, it involved the students of the subject «Anatomical and physiological bases of movement», belonging to the teaching degree of the Universitat Jaume I. On the other hand, there were involved several social organizations which are focused on care for people with mobility difficulties arising from various anatomical and/or physiological conditions. Through this methodology, our students used their knowledge of this subject, to provide a service to the community. Thus, they learned the most relevant contents in a practical and meaningful way, and at the same time they also developed their social and civic skills. The article covers, step by step, the different phases carried out in this service-learning experience implemented in the field of physical education: the design, the practical implementation and the assessment.
\end{abstract}

Key words: Service-Learning, Physical Education Teaching, Methodological Innovation, Functional Diversity, Higher Education.

\section{Introducción}

Como punto de partida entendemos con Aubert, Bizkarra y Calvo (2014) que las actuaciones educativas de éxito en la educación física son aquellas que se orientan a la transformación social. En esta línea, para afrontar el caso de la educación superior nos apoyamos en Díez Gutiérrez (2008) al afirmar que los maestros y maestras del futuro deben adquirir una serie de competencias sociales que les lleven a entender la sociedad y transformarla desde la educación. Así pues, teniendo como horizonte educativo la transformación social y el desarrollo de la competencia social y ciudadana de nuestro alumnado, la experiencia didáctica que se expone en este artículo propone un planteamiento innovador en el ámbito de la educación física universitaria.

En concreto el Área de Didáctica de la Expresión Corporal de la Universitat Jaume I ha diseñado e implementado para la asignatura «Bases anatómicas y fisiológicas del movimiento», un proyecto basado en la metodología de aprendizaje-servicio (en adelante APS), que propone el aprendizaje de contenidos académicos y valores ético-cívicos a través de la prestación de un servicio a la comunidad.

Planteamiento didáctico: APS en la asignatura «Bases anatómicas y fisiológicas del movimiento»

Furco y Billig (2002), Eyler y Giles (1999) y Tapia (2006) coinciden en que el APS es una pedagogía de enseñanza $\quad c \quad u \quad$ y $\quad$ o protagonismo recae en el estudiantado y en la sociedad, siendo el nexo de unión la aplicación de competencias en un contexto real. Asimismo,

Fecha recepción: 07-01-14- Fecha envío revisores: 08-01-14- Fecha de aceptación: 12-02-14 Jesús Gil Gómez.

Av. de Vicent Sos Baynat, s/n

12071 Castelló de la Plana

jegil@uji.es conceptualizado como aprendizaje experiencial, el APS es una metodología pedagógica emergente que propicia al alumnado el aprendizaje significativo de los contenidos curriculares mediante la prestación de un servicio a la sociedad, desarrollando como valor añadido la competencia social y ciudadana (Chiva, 2011; Gil, 2012; Gil, Francisco \& Moliner, 2012). A este planteamiento cabe añadir quela educación física, por su parte, es un área que también contribuye especialmente a la consecución de dicha competencia (Monzonís \& Capllonch, 2014). Así pues, no cabe duda de que unir APS y educación física es una apuesta clara hacia un horizonte educativo propicio para el desarrollo de valores morales y competencias sociales.

Como se desprende de esta conceptualización, la relación entre la educación física y el desarrollo de valores es muy estrecha. En este campo existen diversos trabajos realizados entre los que podemos citar a Prat y Soler (2003), Carranza (2003), Escartí, Pascual y Gutiérrez (2005) y Pinos (2006).

El APS ya ha sido utilizado anteriormente en el ámbito de la formación de docentes de educación física, si bien es cierto que con una extensión todavía limitada. A nivel nacional destacan los trabajos de Ríos $(2003,2009)$ sobre el uso de esta metodología en la integración de discapacitados y de población reclusa. Internacionalmente podemos citar los estudios de Himelein, Passman y Phillips (2010) y MasseySokes y Meaney (2006), quienes realizaron aplicaciones de APS en futuros docentes para actuar sobre la prevención de la obesidad infantil en EEUU; La Master (2001) lo aplicó en escuelas de una zona con graves conflictos sociales y económicos; y Domangue y Carson (2008) lo hicieron realizando actividades físicas para niños y niñas que abandonaron sus hogares y se instalaron en un asentamiento temporal a causa del huracán Katrina. En todos estos estudios se reportan efectos beneficiosos del uso de esta metodología en la formación de futuros docentes de educación física.

En cuanto a la dimensión ético-cívica, la bibliografía muestra indicios de la utilidad de la aplicación del APS en la formación de futuros 
maestros y maestras (Baldwin, Buchanan \& Rudisill, 2007; Meaney, Bohler, Kopf, Hernández \& Scott, 2008; Williams \& Kovacs, 2001).

En la aplicación didáctica que aquí se explica participaron 54 alumnos y alumnas de la asignatura «Bases anatómicas y fisiológicas del movimiento» bajo la guía del profesor titular de la asignatura y el apoyo de otros 2 profesores del área. A través de la misma, el alumnado universitario ha aprendido los conocimientos de la asignatura, prestando un servicio comunitario a personas con dificultades de movilidad derivadas de diversas patologías anatómicas y/o fisiológicas, pertenecientes a diferentes entidades sociales.

El programa ejecutado se ha diseñado de acuerdo con las directrices establecidas por el National Youth Leadership Council (2008) en el documento «Estándares de Aprendizaje-Servicio K-12 para la Práctica de Calidad». Enél, recogiendo la experiencia acumulada por líderes en la aplicación del APS durante 20 años, se establecen ocho criterios prácticos de calidad que se han demostrado eficaces. En la experiencia que presentamos se concretan estos criterios de la siguiente manera:

Ø Duración: se han dedicado 11 semanas, correspondientes a un cuatrimestre académico.

Ø Intensidad: se han invertido una media de 12,3 horas semanales por alumno/a.

Ø Creación de alianzas sociales: se ha interactuado con cuatro entidades de la sociedad castellonense como son el Colegio Público Serrano Suñer, la Asociación Síndrome de Down de Castellón, el Mase de Frater y la Asociación de Diabéticos de Castellón.

Ø Protagonismo del alumnado: se ha fomentado la autonomía de decisión, la capacidad para hacer cambios y la libertad en la forma de exponer y evaluar los resultados.

Ø Desarrollo de la comprensión de la diversidad: el alumnado ha podido conocer las dificultades que presenta el mundo de la discapacidad.

Ø Supervisión del programa: se han establecido varios sistemas de seguimiento como reuniones periódicas grupales y/o del grupo-clase, seguimiento diario con documentos remitidos electrónicamente, y visitas periódicas a las entidades donde se prestaba el servicio.

Ø Reflexión: con ella el alumnado ha podido tomar consciencia de los cambios acaecidos en su estructura cognitiva inicial.

Una vez integrados estos criterios o directrices de calidad, para la secuenciación del programa se ha optado por las tres fases que sugirió Escámez (1990) en relación a la estructuración de programas educativos.

\section{Fase Inicial}

Es la fase de planificación del proyecto y coincide con la denominada Fase de Planificación o Preparación de un programa de APS (Puig, Batlle, Bosch \& Palos, 2007; Puig, 2008; Puig, Martín \& Batlle, 2008; RMC Research Corporation, 2006). Esta fase implica las siguientes tareas:

Descripción de los principios básicos del programa; búsqueda de entidades colaboradoras; establecimiento de contacto con las mismas y con los responsables de acoger alumnado, determinación de responsabilidades en su elaboración, aplicación y evaluación; fijación de su duración e intensidad; delimitación de trabas administrativas y soluciones a las mismas; establecimiento de horarios; creación de grupos de trabajo y asignación de entidades; normas de funcionamiento, y tareas a realizar

Análisis del contexto y diagnóstico por parte de cada grupo de la situación inicial: características de las entidades, del alumnado receptor del servicio, pactos de funcionamiento con los responsables de las entidades, dificultades, recursos, detección de necesidades específicas en la población escogida.

Definición de objetivos y metodologías a aplicar por los grupos de trabajo que debe orientar la elección de estrategias y actividades a realizar adaptadas a las necesidades detectadas.

Delimitación de la línea de actuación de cada grupo de alumnos y alumnas en lo referente a sus relaciones con los agentes educativos implicados (profesor, profesional de la entidad, familias, etc.) y los ámbitos educativos (escolar, entidad, universidad, etc.).

Delimitación por el profesor de los contenidos curriculares a traba- jar en la prestación del servicio, sirviendo como orientación en la determinación de las actividades a desarrollar por cada grupo.

\section{Fase Central}

Aplicación del programa diseñado y prestación del servicio en las entidades seleccionadas. Al corresponderse con la modalidad de servicio directo, el alumnado está en contacto directo con los receptores del servicio. El APS supone trabajo cooperativo, estructurándose la aplicación consecuentemente en grupos de 4-5 personas.

Esta fase coincide con la denominada Fase deAcción en los programas de APS (Puig, Batlle, Bosch \& Palos, 2007; Puig, 2008; Puig, Martín \& Batlle, 2008; RMC Research Corporation, 2006).

\section{Fase Final}

Esta fase incluye las siguientes tareas:

ØDemostración. El alumnado debe demostrar qué aprendizajes ha adquirido transmitiéndolos a la comunidad.

Ø Reflexión. Es una tarea fundamental. No sólo se aprende de la acción, sino también de la reflexión llevada a cabo sobre la propia acción. En los proyectos de APS la reflexión constituye un aspecto central para garantizar el aprendizaje que se deriva de la acción. La reflexión es el marco en el que se sintetiza y reorganiza toda la información que el alumnado recibe de la experiencia.

$\varnothing$ Reconocimiento. Reconocer el valor del trabajo realizado por el alumnado constituye un aspecto básico del proyecto de servicio.

Ø Evaluación. Es diseñada y llevada a cabo conjuntamente por el profesorado y el estudiantado. Se trata de llevar a cabo un análisis del proyecto, dejando constancia del número de participantes, duración, descripción, objetivos curriculares tratados en su desarrollo, beneficios académicos derivados, beneficios afectivos, impacto del proyecto sobre la comunidad (a través de encuestas de opinión, entrevistas, etc.). Se trata de que el alumnado se vaya entrenando en tareas de evaluación, en la síntesis y análisis de la información derivada de la puesta en marcha del proyecto, y comprenda que la evaluación cumple una importante función para la mejora continua de las iniciativas.

$\varnothing$ Seguimiento. En la aplicación de un programa de APS es importante que las conclusiones sobre el mismo las hagan los responsables (en nuestro caso, el profesorado), si bien es conveniente tener en cuenta el criterio valorativo de los profesionales de las entidades que han colaborado. De ahí la necesidad de realizar un seguimiento minucioso de la aplicación del programa, a partir del cual los responsables pueden hacer una valoración de las dificultades, problemas o aciertos del mismo. Por todo ello, consideramos necesario el contacto directo con las entidades, profesionales y alumnado a través de reuniones de seguimiento periódicas.

Metas del programa de APS en la asignatura «Bases anatómicas y fisiológicas del movimiento»

Esteproyecto deAPS implica dos tipos de objetivos: los curriculares y los centrados en la prestación del servicio comunitario. En cuanto al aprendizaje curricular de la asignatura, el uso de APS facilita el trabajo por competencias, dado que supone una aplicación práctica de los contenidos en un contexto real de actuación. Esta es una de las oportunidades que esta metodología brinda y que debe ser aprovechada. Destaca especialmente su idoneidad para el desarrollo de competencias genéricas, destacando las relacionadas con la formación ético-cívica. En nuestro caso, este potencial se concreta en la sensibilización del alumnado universitario frente a los colectivos de personas con dificultades de movilidad derivadas de patologías anatómicas y/o fisiológicas.

Por otra parte, los objetivos del servicio radican en la satisfacción de las necesidades detectadas en dichos grupos, consistentes esencialmente en el desarrollo de las posibilidades motrices de los receptores.

Así pues, se muestran a continuación las metas que persigue el proyecto, tanto a nivel de adquisición de competencias por parte del alumnado, como en lo relativo a los objetivos centrados en el servicio prestado: 
Competencias específicas de la asignatura

Ø Aplicar conocimientos anatómicos y fisiológicos en contextos reales del campo profesional de la educación física.

ØSaber utilizarlos conocimientos académicos relacionados con las bases anatómicas y fisiológicas del movimiento en situaciones concretas, adecuándolos a dichas exigencias.

Ø Ser capaz de poner en juego los conocimientos adquiridos en un amplio espectro de situaciones educativas.

ØCompetencias relacionadas con la formaciónético-cívica

$\varnothing$ Potenciar la sensibilidad ante los colectivos de personas con dificultades en la movilidad derivadas de patologías anatómicas y/o fisiológicas.

Ø Ser capaz de trabajar cooperativamente en entornos reales de aprendizaje.

$\varnothing$ Aplicar habilidades sociales encaminadas a la mejora de la interacción personal.

$\varnothing$ Responsabilizarse del trabajo realizado y de sus consecuencias.

Ø Reconocer la satisfacción y agradecimiento que su trabajo reporta a los receptores del servicio.

ØAplicar las competencias profesionales y personales en un contexto real.

$\varnothing$ Ser capaz de conocerse a uno mismo y tomar conciencia de las propias posibilidades y limitaciones.

Objetivos centrados en el servicio

Ø Mejorar la calidad de vida de las personas con estas dificultades.

$\varnothing$ Reconducir su proceso de desarrollo y aprendizaje motor.

$\varnothing$ Contribuir al desarrollo socio-afectivo de los receptores del servicio.

$\varnothing$ Acercar el mundo de la actividad física y sus ventajas y aplicaciones al colectivo de afectados por las dificultades motrices.

\section{Contexto y agentes implicados en el programa}

En la aplicación de todo programa deAPS confluyen necesariamente diferentes colectivos implicados. Éstos derivan de las vertientes intrínsecas a la propia metodología, a saber, los estudiantes por una parte y las entidades receptoras del servicio por otra. A continuación se describen los principales rasgos de cada uno de los principales grupos implicados en este programa.

En cuanto al grupo de estudiantes, conviene recordar que se trata de alumnos universitarios matriculados en la asignatura «Bases anatómicas y fisiológicas del movimiento», perteneciente a la carrera de magisterio de la Universitat Jaume I.Para más señas puede decirse que por las características de la asignatura, en el momento de aplicación del programa,estos estudiantes deben manejar con suficiencia conocimientos sobre los principales conceptos anatómicos y fisiológicos relacionados con el aparato locomotor. En concreto, los bloques de contenidos y el temario de prácticas sobre el que se apoya la aplicación del APSse detallan en la Tabla 1.

Por otra parte, las entidades en las que el alumnado prestó el servicio dentro de esta aplicación de la metodología APS forman parte del entramado social de la ciudad de Castellón. Todas ellas tienen como nexo común su dedicación a la promoción integral depersonas con algún tipo de dificultad derivada de patologías anatómicas y/o fisiológicas. Brevemente las describimos a continuación:

Tabla 1. Descripción básica de la asignatura "Bases anatómicas y fisiológicas del movimiento". (Guía Docente Oficial de la titulación)

\begin{tabular}{|c|c|}
\hline Bloques temáticos & Temario de prácticas \\
\hline $\begin{array}{l}\text { 1- Generalidades. } \\
\text { 2- Si stemática del ejercicio. } \\
\text { 3-Osteología. } \\
\text { 4-Artrología. } \\
\text { 5- Miología. } \\
\text { 6- Si stema circulatorio. } \\
\text { 7- Met abolismo energético y ATP. } \\
\text { 8- Primeros auxilios. }\end{array}$ & $\begin{array}{l}\text { - Análisis práctico de la estructura y funcionamiento de los } \\
\text { diversos sistemas corporal es. } \\
\text { - Análisis de las posibilidades de movi miento de las } \\
\text { diferentes articulaciones. } \\
\text {-Análisis y determinación de pautas de movimiento } \\
\text { correctas en función de la articulación. } \\
\text { - Interpretación sistemática demovimientos. }\end{array}$ \\
\hline
\end{tabular}

Maset de Frater

El Centro Maset de Frater (Fraternidad Cristiana de Personas con
Discapacidad de Castellón), se constituyó en el curso 1985/1986 para tratar de dar respuesta a las necesidades concretas de un grupo de jóvenes, en su mayoría afectados por parálisis cerebral. Se trata de una experiencia de hábitat, convivencia y formación alternativas a las residencias tradicionales, así como a los centros ocupacionales convencionales. Nuestro alumnado trabajó con un grupo de personas adultas con discapacidad física y/o intelectual, con el objetivo de mejorar sus capacidades motrices, de coordinación, de equilibrio, etcétera. Todos ellos aspectos esenciales para normalizar en la medida de sus posibilidades su vida cotidiana. El trabajo de campo contó con la ayuda del psicopedagogo y la fisioterapeuta del centro, que asesoraron al alumnado en la aplicación de este programa relacionado con la actividad física.

\section{Fundación Síndrome de Down de Castellón.}

Esta entidad, que se nutre principalmente de fondos públicos, atiende a personas afectadas por distintas patologías y a sus familias procedentes de distintas poblaciones de la provincia de Castellón. La Fundación tiene como objetivos fundamentales la promoción y realización de cuantas actividades contribuyan a la mejora de la calidad de vida de las personas con Síndrome de Down y de aquellas con otro tipo de discapacidad intelectual, favoreciendo así su integración social (formación, empleo, recursos sociales, ocio, etc.). El trabajo de campo fue supervisado y contó con la colaboración de dos psicopedagogas de esta asociación.

\section{Asociación de Diabéticos de Castellón (ADICAS).}

Esta entidad, constituida en 1996, persigue movilizar a la sociedad castellonense frente a la diabetes. La asociación tiene como finalidad la defensa de los derechos de estos pacientes. Los grupos de alumnos trabajaron estrechamente con la presidenta de esta asociación, una enfermera especializada en diabetes. Pusieron en funcionamiento un taller de realización de actividades físicas como elemento de control de los niveles de glucosa en la sangre. Los participantes en este taller, que se llevó a cabo en la sede social de la asociación y también al aire libre, fueron personas con esta patología, todos ellos en edad adulta.

\section{Colegio Público Serrano Súñer de Castellón.}

Se trata de un centro escolar de titularidad pública que imparte la docencia de las etapas de infantil y primaria. Está ubicado en un barrio de clase social media-baja, con una alta concentración de personas inmigrantes, problemas socio-económicos evidentes, fuerte deterioro urbanístico y una falta innegable de algunos servicios públicos básicos. A nivel educativo este centro cuenta con una unidad de atención a alumnado con discapacidades motoras diversas, agravadas en algunos casos por ciertas deficiencias de carácter psíquico. Esta población escolar con dificultades manifiestas es atendida por tres fisioterapeutas a tiempo completo, que nunca llegan a cubrir totalmente las necesidades de rehabilitación que estos alumnos requieren, por lo que se hallan desbordados en muchos aspectos. Nuestros estudiantes realizaron tareas de apoyo a estos profesionales y también, en otro segmento horario y supervisados por otras personas del equipo docente, realizaron tareas de atención a estos colectivos escolares con dificultades.

\section{Plan de trabajo establecido y actividades efectuadas}

Una vez descritos las características de nuestro alumnado, así como las de la asignatura y las de las entidades colaboradoras sobre las que pivota el proyecto, pasamos a describir una a una las fases llevadas a cabo en este programa de APS, de acuerdo con el planteamiento didáctico expuesto previamente.

\section{Fase inicial (preparación o planificación).}

Tras un primer período en el que se impartieron los contenidos teóricos de la asignatura, apoyándose este proceso en prácticas de aula y medios audiovisuales, la fase de aplicación de los mismos (interpretación sistemática de movimientos, ejes, planos, tipos de contracción muscular, actuación de las articulaciones en función de su tipo y posibi- 
lidades de movimiento, análisis de la intervención muscular en los movimientos, etc.) fue llevada a cabo a través de la metodología APS.

Dada la especificidad de los contenidos, se buscaron entidades en las cuales se trabajara con personas que presentaran deficiencias en cuanto a su movilidad, para que nuestro alumnado pudiera proponer patrones de movilidad articular activa y pasiva, así como de potenciación muscular, utilizando las propiedades de contracción de la musculatura (isométrica, isotónica concéntrica e isotónica excéntrica), de forma que las sesiones a impartir les ayudaran a mejorar aspectos de su movilidad limitada (equilibrio, fuerza, flexibilidad, coordinación, etc.). Se contactó con entidades de estas características, confirmando que bien por la cantidad de personas con dificultades, por la falta de profesionales en la materia o por falta de recursos, la atención que se ofrecía atendía una necesidad palpable. Es decir, se detectó una necesidad social no atendida y de gran importancia para los implicados.

Se estableció contacto con las cuatro entidades expuestas en el apartado anterior. Todas ellas tenían como característica común trabajar con personas con movilidad reducida y en riesgo de exclusión social por motivos asociados a sus patologías.

A continuación se presentó el proyecto de APS a los responsables de las entidades, explicando que consistía en la realización de sesiones prácticas en las que los estudiantes universitarios trabajarían directamente con sus usuarios, con el objetivo de mejorar aspectos básicos de su movilidad, aplicando y aprendiendo los contenidos básicos de la asignatura.

Se estableció el acuerdo con las cuatro entidades, siguiendo las especificidades de cada una de ellas. Cabe resaltar el compromiso de implicación en el proyecto de los profesionales, que fueron los encargados de acoger en sus sedes al alumnado de la universidad.

\section{Fase central (acción).}

Acabada la planificación, se realizó la fase que implica el servicio directo en las entidades. En este punto conviene resaltar la importante interacción entre el alumnado y los receptores de la prestación, así como la gran cantidad de feedback que recibieron nuestros estudiantes, no sólo a nivel de contenidos sino también en la dimensión humana.

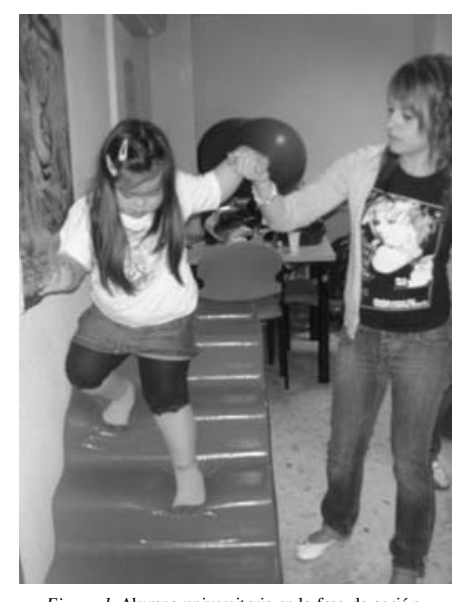

La principal característica de esta fase a nivel de ejecución del programa fue el seguimiento del trabajo que se realizó por parte del profesorado. Este seguimiento se hizo mediante las horas de tutoría correspondientes, la presentación diaria de una serie de documentos de seguimiento, las visitas del profesorado a las entidades receptoras y las reuniones de éste con los profesionales responsables de las mismas.

\section{Fase final.}

En esta fase se realizó la valoración del programa, que incluye las subfases de demostración, reflexión, reconocimiento y evaluación.

La demostración de los aprendizajes adquiridos se hizo de diversas maneras. Al ser un servicio directo y tener que trabajar con personas con necesidades especiales que requerían una gran atención, los aprendiza-

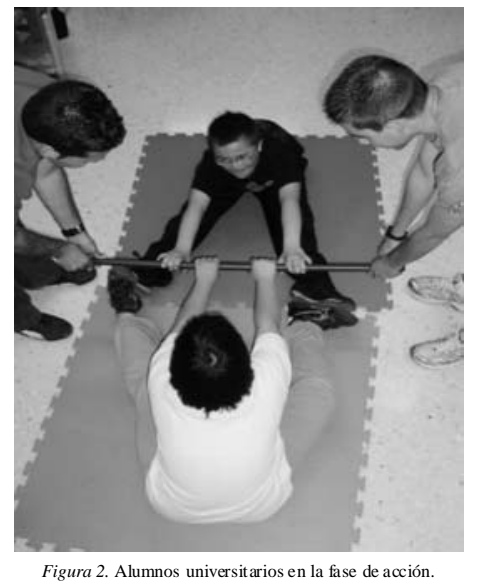

jes se demostraban en cada sesión, ya que los receptores del servicio iban retroalimentando la actividad realizada por nuestros estudiantes. Si la sesión estaba bien hecha y era atractiva eran los mismos receptores los que ejercían el feedback, con lo que el alumnado de la universidad estaba continuamente demostrando los conocimientos aprendidos. Además, al ser un trabajo tan específico en el sentido de requerir una perfecta aplicación de los ejercicios, fueron principalmente los profesionales de las entidades quienes supervisaron el proceso. Asimismo, se realizó una exposición pública de cada grupo ante la totalidad del grupoclase, en la que se explicaron todos los aspectos de la actividad: objetivos educativos, contenidos aprendidos, tipo de actividades diseñadas para responder a las necesidades físicas detectadas, explicar los ejercicios diseñados para trabajar la movilidad articular, el análisis de los ejercicios en base a la intervención muscular, así como también aspectos de interacción con las personas con necesidades especiales, etc.).

Por otra parte, la reflexión fue un tema crucial en la valoración del proyecto APS. Como hemos argumentado previamente, no puede haber una aplicación APS que no tenga una fase de reflexión, entendida como la forma de hacer que el alumnado interiorizara tanto los aprendizajes académicos, como también los valores que se han puesto en juego en esta aplicación metodológica. En nuestro caso la reflexión se realizó teniendo como referencia las herramientas e instrumentos que se muestran en los apéndices.

En cuanto a la fase de reconocimiento, que el alumnado sintiera que su trabajo estaba siendo apreciado por los receptores era necesario para valorar el alcance de su actuación. La simple retroactividad que produce el contacto directo y físico (recordemos que debían manipular articulaciones limitadas en su movimiento, músculos atrofiados y/o ayudar físicamente a personas para poder realizar los ejercicios) con este tipo de personas y conocer sus historias, hizo finalmente que el alumnado percibiera como un hecho útil el trabajo realizado.

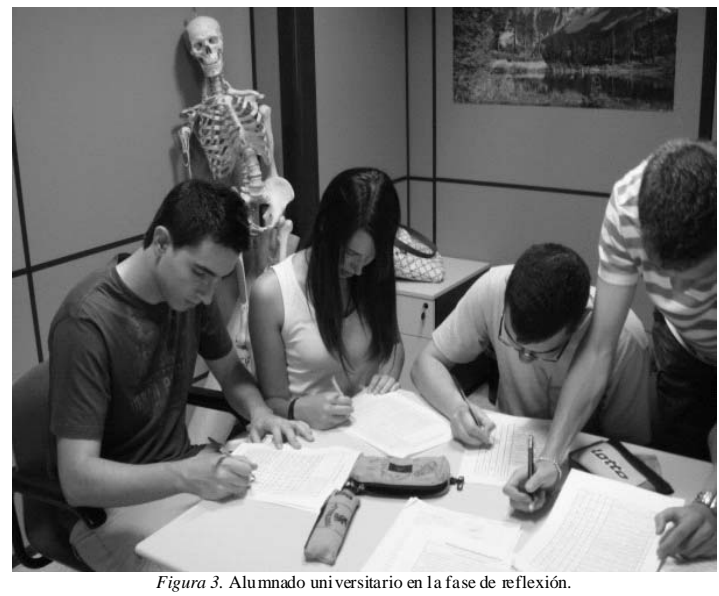




\section{Resultados}

Los resultados de una experiencia de APS pueden interpretarse desde dos perspectivas distintas: atendiendo al proceso de enseñanza/ aprendizaje del estudiantado universitario, y poniendo la mirada en la valoración y análisis general del proyecto, dando voz en este caso a todas las partes implicadas.

En cuanto a la valoración del proceso de enseñanza/aprendizaje, se ha optado por un enfoque en el que la «racionalidad práctica o ética» planteada por López, Monjas, Gómez, López, Martín, González et al. (2006), debe centrar el foco del proceso evaluativo a partir de un diseño basado en procesos formativos, compartidos y dialógicos. Asimismo, este planteamiento debe ser reinterpretado en nuestro caso, dadas las diferencias a tener en cuenta para el contexto universitario. En cualquier caso los cambios metodológicos marcados por el Espacio Europeo de Educación Superior (EEES) también implican necesariamente una reflexión y ajuste del proceso evaluativo del alumnado en el mismo sentido. Por ello, de acuerdo con los estudios de Fernández (2006) y Palomares y Garrote (2009), hemos enfocado nuestro planteamiento docente hacia la aplicación y resolución de problemas lo más reales posibles. En este sentido, una evaluación acorde a las demandas pedagógicas del EEES debe orientarse a poner el énfasis sobre el papel activo y participativo del alumnado, así como a dar mayor importancia a la finalidad formativa de la evaluación (Doménech, 2011). De un modo concreto ello implica la necesidad de atender aspectos como: la existencia de feedback durante el proceso de enseñanza-aprendizaje, la posibilidad de mejora durante el propio proceso, la autoevaluación y evaluación entre compañeros de forma que se fomente la responsabilidad y la autonomía, así como un serio proceso de reflexión en búsqueda de aplicar los conocimientos adquiridos de la manera más creativa posible para resolver problemas reales (Villardón, 2006).

Este hecho toma más relevancia, si cabe, cuando nuestro propósito se centra en formar a futuros/as docentes. En el caso de la educación superior, a nuestro juicio, el foco de evaluación de los futuros maestros y maestras debe situarse en la adquisición de habilidades y competencias docentes aplicadas al área en cuestión. Así pues, es razonable cimentar el proceso evaluativo del proyecto deAPS en base a la observación directa de nuestros alumnos y alumnas en el desempeño de su rol de manera práctica, así como en base a la reflexión y el diálogo posterior. Además, es sabido que el estudiante enfoca su aprendizaje según percibe que va a ser evaluado, por lo que una evaluación lo más aplicada, global y holística posible redundará en un aprendizaje del mismo carácter (Doménech, 2011).

Por todo ello, para evaluar nuestro programa de APS hemos realizado una valoración conjunta del programa en la que han intervenido los propios estudiantes, el profesor y los representantes de las entidades implicadas. El profesorado ha mantenido reuniones con los profesionales responsables en las que se ha valorado la actividad globalmente, se han atendido sugerencias, se ha examinado el nivel de aprendizajes adquirido a nivel práctico y se han revisado las conductas manifestadas por el estudiantado en el trato con personas limitadas motrizmente. Posteriormente se ha realizado una sesión entre el profesorado y cada grupo, en la que se ha ido revisando toda la documentación elaborada, comentando sus conclusiones sobre esta forma de aprender y sobre las vivencias experimentadas en el contacto con colectivos en riesgo de exclusión. Finalmente, se ha hecho una puesta en común sobre la opinión de los expertos y su visión de los aprendizajes adquiridos.

Después de todo este proceso, los resultados obtenidos en lo referente a los contenidos específicos de la asignatura muestran que la adquisición de conocimientos es equiparable a los obtenidos por alumnado de cursos anteriores. No obstante, cabe destacar en este sentido la manifestación explícita del propio alumnado sobre la riqueza y motivación que la experiencia ha significado para ellos. Una aceptación que indudablemente revierte en la significatividad de los aprendizajes logrados.

Asimismo, de acuerdo con la conceptualización delAPS, es necesario valorar también los aprendizajes del alumnado a nivel de valores ético-cívicos. En este punto los resultados de los instrumentos emplea- dos muestran que se han desarrollado actitudes de ayuda y colaboración, así como de respeto por las normas sociales, y de responsabilidad y sensibilidad social, mostrándose estas categorías en sintonía con los resultados obtenidos por Baldwin, Buchanan y Rudisill (2007), Meaney, Bohler, Kopf, Hernández y Scott (2008) y Williams y Kovacs (2001).

Por otra parte, como se comentaba al inicio de este apartado, para ofrecer una valoración general del proyecto es oportuno dar voz a todas las partes implicadas. Se implementó a tal efecto un sistema de diálogo constante con los responsables de las entidad espara evaluar el funcionamiento y reajustar el proceso. De acuerdo con Puig, Batlle, Bosch y Palos (2007), Puig (2008), Puig, Martín y Batlle(2008) y RMC Research Corporation (2006) el APS debe concluir con una fase de reconocimiento. En nuestro caso se organizó en la universidad la «I Jornada de Aprendizaje-Servicio: experiencias en el ámbito de la Educación Física». Fue una jornada abierta en la que intervinieron todos los grupos de alumnos, las entidades participantes, el profesorado y los profesionales implicados. En ella se valoró el funcionamiento del proyecto y las dificultades y beneficios del mismo.

\section{Conclusiones}

La experiencia nos permite concluir que el APS en el ámbito de la educación física es una metodología adecuada para desarrollar competencias generales y específicas. Permite la movilización de capacidades y aprendizajes que deben dar respuesta a exigencias y necesidades reales, por lo que es una magnífica oportunidad para la renovación pedagógica que plantea elEEES.

Por otra parte, es evidente que el empleo del APS supone dificultades añadidas a los usos metodológicos tradicionales en términos de responsabilidad, logística, organización y gestión de la docencia. La apertura de la docencia fuera de las aulas y su contextualización en escenarios sociales reales, implica la existencia de nuevos flancos de actuación que hacen que el proceso formativo sea ineludiblemente más complejo. Sin embargo, como contrapunto, el APS aporta como valor añadido un aumento de la motivación y la significatividad del aprendizaje, así como nuevas posibilidades de crecimiento personal.

Finalmente, en pos de superar las dificultades relatadas en esta experiencia, así como en la bibliografía referida, es deseable que en futuras aplicaciones se abunde en la investigación de los efectos y las posibilidades que el APS proporciona en la educación física. Algunas líneas que desde aquí se sugieren son: el estudio de los efectos que produce el APS en el alumnado; las implicaciones que acarrea en la gestión de la docencia; los efectos sobre los colectivos, entidades o comunidades receptoras del servicio; y la creación y consolidación de redes para compartir experiencias, conocimientos e investigaciones.

\section{Referencias}

Aubert, A., Bizkarra, M., \& Calvo, J. (2014). Actuaciones educativas de éxito desde la Educación Física. RETOS. Nuevas tendencias en Educación Física, Deporte y Recreación, 25, 144-148.

Baldwin, S. C., Buchanan, A. M., \& Rudisill, M. E. (2007). What Teacher Candidates Learned about Diversity, Social Justice, and Themselves from Service-Learning Experiences. Journal of Teacher Education, 58(4), 315-327.

Carranza, M. (2003). Educación Física y valores; educando en un mundo complejo: 31 propuestas para los centros escolares. Barcelona: Graó.

Chiva, O. (2011). Aprendizaje-servicio: una apuesta clave para la consecución de las ciudades educadoras. Quaderns digitals. Actas Congreso Mejora Educativa y Ciudadanía Crítica. Universitat Jaume I. (En línea). Recuperado el 20/06/2013 de w w w. q u a d e r n s d i g i t a l s . n e t/

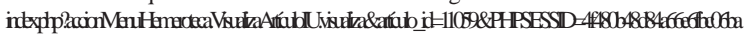
Díez, E. J.(2008). El modelo neoliberal de convergencia europea en la formación superior. Tándem. Didáctica de la Educación Física, 26, 7-18.

Domangue, E., \& Carson, R. (2008). Preparing Culturally Competent Teachers: Service-Learning and Physical Education Teacher Education. Journal of Teaching in Physical Education, 27(3), 347-367.

Doménech, F. (2011). Evaluar e investigar en la situación educativa universitaria. Un nuevo enfoque desde el Espacio Europeo de Educación Superior. Castelló de la Plana: publicacions de la Universitat Jaume I.

Escámez, J. (1990). Drogas y escuela. Una propuesta de prevención. Madrid: Dykinson.

Escartí, A., Pascual, C., \& Gutiérrez, M. (2005). Responsabilidad personal y social a través de la Educación Física y el deporte. Barcelona: Graó. 
Eyler, J., \& Giles, D. E., Jr. (1999). Where's the learning in service-learning? San Francisco: Jossey-Bass.

Fernández, A. (2006). Metodologías activas para la formación de competencias. Educatio siglo XXI, 24, pp. $35-56$

Furco, A., \& Billig, S.H. (2002). Service Learning. The essence of the Pedagogy. Greenwic. C.T.

Gil, J. (2012). El aprendizaje-servicio en la enseñanza superior: una aplicación en el ámbito de la educación física. Tesis Doctoral. Universitat Jaume I de Castellón.

Gil, J., Francisco, A., \& Moliner, L. (2012). La educación física y el aprendizaje servicio: abriendo el entorno natural a la escuela. Tándem, Didáctica de la Educación Física, 38, 95-100.

Himelein, M., Passman, L., \& Phillips, J. M. (2010). College Teaching and Community Outreaching: Service Learning in an Obesity Prevention Program. American Journal of Health Education, 41(6), 368-378.

LaMaster, K. J. (2001). Enhancing Preservice Teachers Field Experiences through the Addition of a Service-Learning Component. Journal of Experiential Education, 24(1), 27-33.

López, V. M.; Monjas, R.; Gómez, J.; López, E. M.; Martín, J. F.; González, J.; Barba, J. J.; Aguilar, R.; González, M.; Heras, C.; Martín, M. I.; Manrique, J.C.; Subtil, P.; Marugán, L. (2006). La evaluación en educación física. Revisión de los modelos tradicionales y planteamiento de una alternativa: la evaluación formativa y compartida. RETOS. Nuevas tendencias en Educación Física, Deporte y Recreación, 10, 31-41.

Massey-Sokes, M., \& Meaney, K. S. (2006). Understanding Our Service-Learning Community: An Exploratory Study of Parent, Teacher, and Student Perceptions about Childhood Obesity. Health Educator, 38(2), 53-60.

Meaney, K. S., Bohler, H. R., Kopf, K., Hernandez, L., \& Scott, L. S. (2008). ServiceLearning and Pre-Service Educators' Cultural Competence for Teaching: An Exploratory Study. Journal of Experiential Education, 31(2), 189-208.

Monzonís, N., \& Capllonch, M. (2014). La educación física en la consecución de la competencia social y ciudadana. RETOS. Nuevas tendencias en Educación Física, Deporte y Recreación, 25, 180-185.

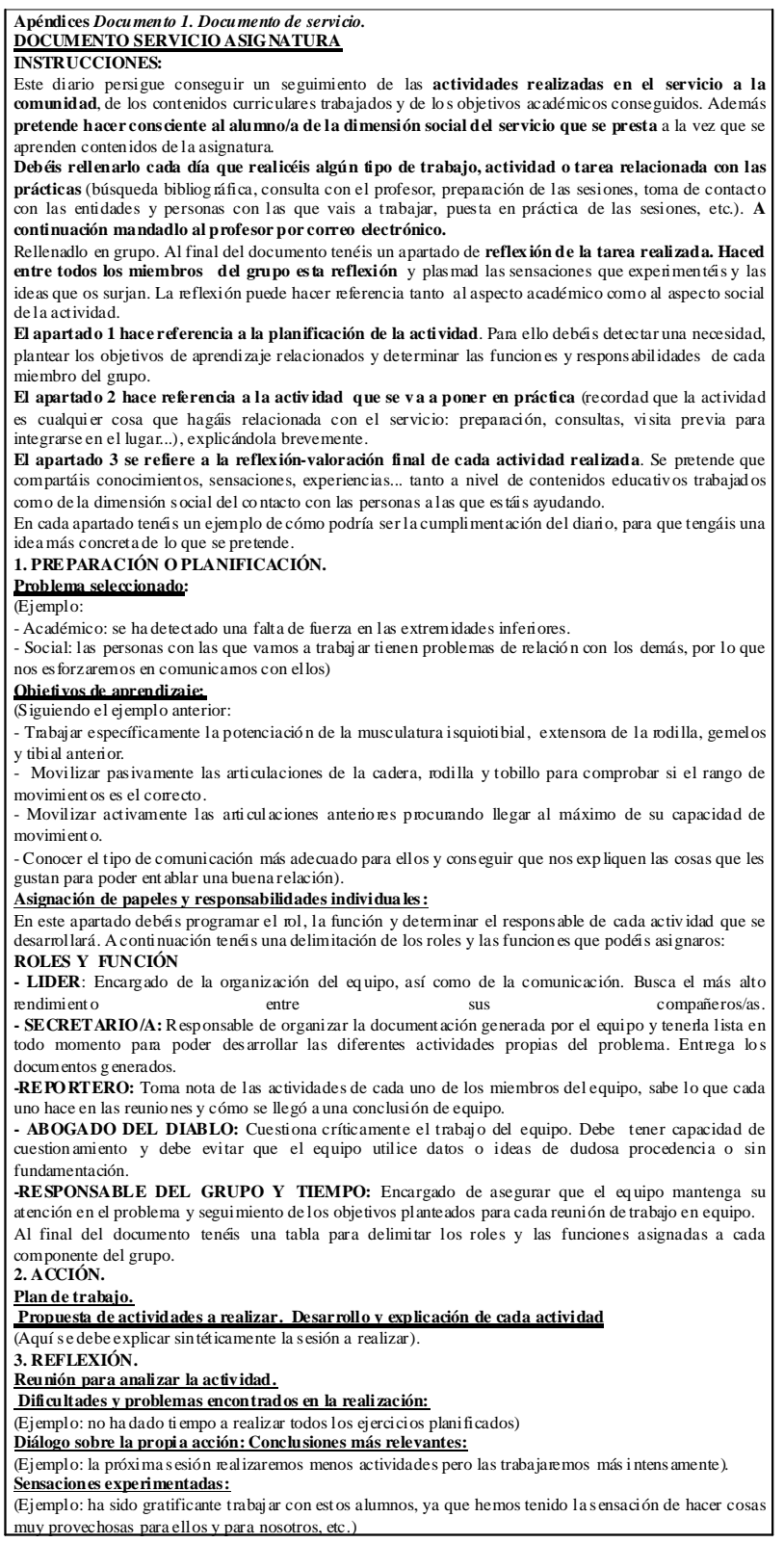

National Youth Leadership Council (2008). K-12 Service-Learning Standards for Quality Practice. Recuperado el 12/02/2010 de: http://nylc.org/sites/nylc.org/files/files/ Standards Oct2009-web.pdf.

Palomares, A., \& Garrote, D. (2009). Un nuevo modelo docentepory para el alumnado, ENSAYOS, Revista de la Facultad de Educación de Albacete, 24, 25-34.

Pinos, M. (2006). Un mundo de alternativas: proyecto integral de educación en valores a través de la Educación Física. Ministerio de Educación y Ciencia. Centro de Investigación y Documentación Educativa.

Puig, J. M., Batlle, R., Bosch, C., \& Palos, J. (2007). Aprendizaje servicio. Educar para la ciudadanía. Barcelona: Ministerio de Educación y Ciencia, Octaedro.

Puig, J.M. (2008). Com fer APS en les entitats socials? (En línea). Recuperado el 15/ 01/2010 de: http://www.aprenentatgeservei.cat/intra/aps/documents/ aps entitats socials3.pdf.

Puig, J.M., Martín, X., \& Batlle, R. (2008). Com començar una experiència d'APS? Recuperado el 15/01/2010 de: http://www.aprenentatgeservei.org/intra/aps/documents/ Guia_com_comencar_REVISADA.pdf.

Prat, M., \& Soler, S. (2003). Actitudes, valores y normas en la Educación Física y el Deporte. Reflexiones y propuestas didácticas. Barcelona: Inde.

Ríos, M. (2003). El juego y el alumnado con discapacidad intelectual y/o plurideficiencia.Tándem: Didáctica de la Educación Física, 4(11), 40-49.

Ríos, M. (2009). La Educación Física adaptada a pacientes con trastorno mental severo: una experiencia de aprendizaje servicio. Tándem: Didáctica de la Educación Física, 8(29), 79-90.

RMC Research Corporation (2006). K-12 Service-Learning Project Planning Toolkit. Scotts Valley, CA: National Service-Learning Clearinghouse. (En línea) Recuperado el 3/02/2010 de: http://www.servicelearning.org/filemanager/download/ K-12 Service-Learning Project Planning Toolkit.pdf.

Tapia, M.N. (2006). La solidaridad como pedagogía: el aprendizaje-servicio en la escuela. Buenos Aires: Ciudad Nueva Editorial. $2^{\mathrm{a}}$ edición.

Villardon, L. (2006). Evaluación del aprendizaje para promover el desarrollo de competencias. Educatio siglo XXI, 24, 57-76.

Williams, K., \& Kovacs, C. (2001). Balance and Mobility Training for Older Adults: An Undergraduate Service-Learning Experience. Journal of Physical Education, Recreation \& Dance, 72(3), 54-58.

\section{Documento 2. Diario de Seguimiento de Servicio}

DIARIO DE SE GUIMIENTO DE SER VICIO

NSTRUCCIONES:

The

Deéis rellenarlo cada día que realicéis algún tipo de trabajo, actividad o tarea relacionada con las Drácticas (búsqueda bibliográfica, consulta con el profesor, preparación de las sesiones, toma de contacto

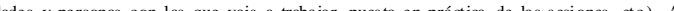
adadlo al profesor por correo electrónico. Rellenadlo en grupo. Al final del documen to tenéis entre todos los miembros del grupo esta reflexión y plasmad las sensaciones que experimentés y las ideas que os surjan.

1. PREPARACIÓN O PLANIFICACIÓN

Problema seleccionado:

2. ACCIÓN

Propuesta de adividades a realizar. Desarrollo y explicación de cada acti vidad:

Aquí se debe explicar sintéticamente la sesiôn o actividad a realizar)

Asignaion de papeles y respons abilidades individuales: (En este cuadro
responsabilidad). REFLEXIÓN

\section{Reunión nara analizar la actividad.}

ficultades y problemas encontrados en la realizació

Tálogo sobre la propia acción. Conclusiones más relevante

Tsadones experimentadas:

\section{Documento 3. Ficha de Control del Servicio Prestado. \\ FICHA DE CONTROL DEL SERVICIO PRESTADO}

SCONSIDERACIONES Servicio (APS). El Aprendizaje de Servicio se convierte en parte integrante de la educación universitaria, con el objeto de in er entar el servicio en el aula y dentro del curriculum académico, ya que considera la

e caracteriza por: 1.- Es un mét odo de trabajo activo: el alumno/a participa en la adquisición del 3.- Estimula el trabajo cooperativo. 4- El profesorado es facilitador'a o tut or/a del aprendizaje.

Tiene como objetivos básions: promover en el alumno a la respons abilidad de su aprendizaje, desarrollar

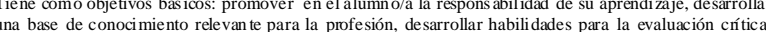
desarrollar habilidades para las relaciones interpersonales, etc

ESTA FICHA DE CONTROL LA DEBÉIS ENTREGAR AL PROFESOR CUANDO ACABE EL SERVICIO, DEBIDAMENTE RELLENADA. MANDADLA POR CORREO ELECTRÓNICO AL FINALIZAR.

DESCRIPCIÓN DEL SERVICIO

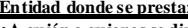

A quién o quienes se dirige?

Duración prevista:

¿Qué se pretende obtener?

\section{CALENDARIO DE REUNIONES DE TRABAJO}

Cualquier actividad que hagais relacionada con este trabajo debe ser anotada en la fich bibliográficas, puesta en práctica del servicio, tut orías con el profesor, búsqueda de información.. 